\title{
Bound states in the continuum in the visible
}

\section{emerging from out-of-plane magnetic dipoles}

\author{
Shunsuke Murai, ${ }^{*, \dagger}$, Diego R. Abujetas, " Gabriel W. Castellanos, ${ }^{\ddagger}$ José A. \\ Sánchez-Gil, ${ }^{*}$ Feifei Zhang, ${ }^{\dagger}$ and Jaime Gómez Rivas*, \\ $\dagger$ Department of Material Chemistry, Graduate School of Engineering, Kyoto University, \\ Nishikyo-ku, Kyoto 615-8510, Japan \\ $\ddagger$ Department of Applied Physics and Institute for Photonic Integration, Eindhoven \\ University of Technology, P.O. Box 513, 5600 MB Eindhoven, The Netherlands \\ IInstituto de Estructura de la Materia (IEM-CSIC), Consejo Superior de Investigaciones \\ Cientificas, Serrano 121, 28006 Madrid, Spain
}

E-mail: murai@dipole7.kuic.kyoto-u.ac.jp; j.sanchez@csic.es; j.gomez.rivas@tue.nl

\begin{abstract}
Bound states in the continuum (BICs) are electromagnetic modes with a dispersion inside the light cone and infinite lifetimes. This exceptional property has led to intensive research and the demonstration of BICs in the gigahertz, teraherz, near-infrared, up to the visible region. In this study, we design and experimentally demonstrate optical BICs using a sub-diffraction lattice of Si nanodisks. The out-of-plane magnetic dipole resonance in the dielectric nanodisks couples with the sub-diffraction lattice and defines a symmetry-protected BIC at normal incidence. This mode becomes a quasi-BIC as the angle of incidence is increased. The spectral position of the BIC can be controlled with the diameter of the nanodisks, which governs the out-of-plane magnetic dipole. The investigated BIC is robust to shape irregularities of the individual nanodisks. This


robustness makes the design strategy of the BICs presented here very attractive for applications in which high fabrication precision can not be achieved.

Keywords: bound states in the continuum, dielectric metasurfaces, silicon nanoparticles.

\section{Introduction}

Dielectric metasurfaces are thin nanophotonic platforms to modulate light propagation via the manipulation of light phases using arrays of scattering elements or meta-atoms. Because dielectric meta-atoms, such as nanoparticles, can support both electric dipole (ED) and magnetic dipole (MD) resonances, elaborated design of meta-atoms to tune the interference between dipoles results in exotic optical effects. Examples of these effects are the Kerker effect or zero-backscattering, ${ }^{1-12}$ surface lattice resonances, ${ }^{13-15}$ and non-radiating anapole modes. ${ }^{16}$ Of particular interest is the emergence of optical bound states in the continuum (BICs), which are dark modes non-excitable from outside. ${ }^{17-20}$ In contrast to defect states in photonic crystals, which are isolated energy levels spatially localized in the defect, BICs are extended eigenstates in the system that coexist in the same spectrum with a continuum of radiating waves that can carry energy away. Several configurations have been proposed to support BIC modes, including photonic crystals and metasurfaces, ${ }^{21-29}$ gratings, ${ }^{30}$ corrugated nanowires, ${ }^{31}$ and waveguides. ${ }^{32-34}$ BICs have the unique property as cavities that are isolated from far-field perturbations, but they are spatially extended in the system. This character makes BICs particularly attractive to study light-matter interaction, and to enhance and modify the properties of matter. ${ }^{27,35}$ Light-matter interaction has been extensively explored using various cavities in the visible, but the experimental realization of BICs is relatively difficult compared to lower frequency regions due to material limitations. High refractive index materials (HRIs), such as Si and GaAs are lossless in near infrared, but they have finite absorption in the visible, which smears out the BICs. Therefore, a design strategy is required that is robust enough to realize (quasi-) BICs under optical losses. 
In this manuscript, we demonstrate BICs in the visible region using nanodisk arrays made of polycrystalline silicon. We use quasi-analytical calculations based on a coupled electric/magnetic dipole theory (CEMD) to design the nanodisk geometry and array periodicity. This design strategy allows the decoupling of out-of-plane MD in the nanodisks from in-plane modes, the former thus becoming a symmetry-protected BIC at normal incidence. At the BIC condition at $\lambda \sim 750 \mathrm{~nm}$, light is trapped in the plane of the array with nanodisk heights as small as $80 \mathrm{~nm}$. This work realizes BICs in metasurfaces in the optical region using the out-of-plane MD. We further discuss the effects of optical losses, i.e., absorption inherent to material, and radiation losses due to shape irregularities in the nanodisks imposed by fabrication.

\section{Designing BICs from vertical magnetic dipoles}

In order to realize BICs in the visible, it is preferable to have a material with high refractive index $(n)$. This material should have a negligible absorption $(k)$. A large $n$ means a large polarizability or strong interaction with light that allows some degree of freedom in the design of the metasurface, while a negligible $k$ is a prerequisite for a long mode lifetime. Compared to other frequency regions, where several semiconductors satisfy this requirement, there is no ideal material with very large $n$ and no $k$ in the visible. This limitation imposes a challenge for the experimental demonstration of BICs in this region of the electromagnetic spectrum. Figure 1a illustrates the values of $n$ and $k$ at the edge of the visible spectrum $(\lambda=750$ $\mathrm{nm}$ ) of representative semiconductors. Some of these materials, such as $\mathrm{Si},{ }^{31,36} \mathrm{GaAs},{ }^{37}$ $\mathrm{GaN},{ }^{38}$ and $\mathrm{Si}_{3} \mathrm{~N}_{4}{ }^{39,40}$ have been used to demonstrate BICs in the visible range. From this figure, we choose $c$-Si because of its balanced $n$ and $k$ values and the compatibility with $\mathrm{Si}$ electronics platforms for future integration. We also note in this figure that $k$ of $c$-Si is much smaller than that of amorphous $\mathrm{Si}(a-\mathrm{Si})$, which has been widely employed in metasurface studies. We demonstrate that absorption severely smears the BICs and using low-loss $c$-Si 
is advantageous to realize BICs in the visible.

The sample consists of a sub-diffraction array of polycrystalline Si nanodisks on a synthetic quartz substrate (see the conceptional image in Fig. 1b). The fabrication procedure of Si nanodisk arrays is described in the Methods section. The individual Si nanodisks are designed to support an out-of-plane MD resonance that is a dark mode at normal incidence. When arranged in the sub-diffraction array, the radiation from the out-of-plane MDs can couple into a mode bounded to the plane of the array, while leakage into vertical directions is cancelled by the vertical of orientation of the MDs. The coupled out-of-plane MDs define a symmetry-protected BIC at normal incidence.

The diameter and height of the fabricated nanodisks are $D=248 \mathrm{~nm}$ and $h=80 \mathrm{~nm}$, respectively. The diameter is designed to spectrally separate the out-of-plane MD from the in-plane MD and ED of the nanodisks. The out-of-plane MD stems from the in-plane circulation current in the nanodisk, while the in-plane MD and ED originate from charge oscillations in the vertical and horizontal directions, respectively. These distinct origins mean that the spectral positions of the resonances can be tuned with the dimensions and geometry of the nanodisks. We choose the diameter and height such that the in-plane MD resonance is at a frequency higher than that of the out-of-plane MD resonance. This tuning is done by decreasing the height and increasing the diameter of the nanodisks. Incidentally, we note that, as seen in the SEM image (Fig. 1c), the nanodisks have a corrugated surface, the effect of which is discussed later in the manuscript. The nanodisks are arranged in a square lattice with a period $a=340 \mathrm{~nm}$. The period is chosen so that the spectral region of interest lies within the sub-diffraction regime, so that the array behaves effectively as a metasurface. 


\section{Results and Discussion}

\section{Dispersion measurements}

We measure the zeroth-order (forward) transmission spectrum by illuminating the sample with a collimated beam from a halogen lamp. To realize a homogeneous medium surrounding the nanodisks that provides out-of-plane symmetry, we place a quartz coverslip on top of the nanodisk array with index matching oil $(\varepsilon=2.1)$ in between. We evaluate the optical extinction $(E)$, defined as $E=1-T / T_{0}$, where $T$ is the transmission through the sample and $T_{0}$ is the transmission through a reference consisting of a substrate, index-matching oil, and superstrate. The extinction spectrum is shown for TE-polarization in Fig. 2a as a function of the angle of incidence, $\theta_{i n}$, where the incident plane is defined as the $z-x$ plane. The white lines and curves in this figure denote the in-plane diffraction conditions or Rayleigh anomalies, satisfying

$$
\vec{k}_{0}=\vec{k}_{x}\left(\theta_{i n}\right) \pm \vec{G}\left(m_{1}, m_{2}\right)
$$

where $\vec{k}_{0}$ is the wave vector of the in-plane diffracted orders, $\overrightarrow{k_{x}}$ is the in-plane component of the wave vector of the incident beam, which depends on the incident angle $\theta_{i n}$, and $\vec{G}\left(m_{1}, m_{2}\right)=2 \pi / a\left(m_{1}, m_{2}\right)$ is the reciprocal lattice vector of the array with $m_{1}$ and $m_{2}$ the orders of diffraction in the $x$ - and $y$ - directions, respectively, and $a$ the lattice constant. Three extinction peaks appear at normal incidence, as seen in Fig. 2b. The peaks at $\lambda=600$ and $640 \mathrm{~nm}$ are ascribed to in-plane $\mathrm{MD}\left(m_{x}\right)$ and ED $\left(p_{y}\right)$ Mie resonances in the nanodisks, respectively, as confirmed by the coupled electric/magnetic dipole (CEMD) calculations presented in the next section. The peak at $\lambda=500 \mathrm{~nm}$ is a higher order resonance and it will not be further discussed. We note that the in-plane MD and ED resonances have different dispersion because of their coupling to different diffraction orders in the array. ${ }^{15}$ For TE polarization, where the incident light is polarized along the $y$-axis, the ED in the nanodisks oscillates in the $y$ - direction. The emission or scattered field by the dipole moment $p_{y}$ is maximum in the orthogonal direction $(x-$ direction $)$, coupling preferentially to the $m_{1}= \pm 1$, 
$m_{2}=0$ diffraction orders as the angle of incidence is changed in the $z-x$ plane. This coupling leads to dispersion of the in-plane ED that can be appreciated in Fig. 2. In contrast, the in-plane MD resonance is non-dispersive, i.e., no change in the resonance wavelength of $600 \mathrm{~nm}$ is observed when the angle of incidence is changed. The dominant electric field component associated to this resonance is along the vertical $z$-direction, which reduces the coupling efficiency to the in-plane diffraction orders.

We focus further on the additional resonance appearing for TE polarization at longer wavelengths and at angles of incidence larger than zero. This resonance is associated to the out-of-plane $\mathrm{MD}\left(m_{z}\right)$, which becomes dark at normal incidence. We investigate in more detail the origin of this resonance in the next section. The extinction spectra measured at different angles visualize the appearance of $m_{z}$ for TE polarization and $\theta_{i n}>6^{\circ}$ (Fig. 2b). These measurements illustrate how the symmetry protected BIC evolves into a quasi-BIC. For TM polarization, the out-of-plane ED $\left(p_{z}\right)$ appears instead of $m_{z}$. However, $p_{z}$ overlaps with the in-plane modes and cannot be spectrally separated (see Supp. Inf. Fig. S1.)

\section{Origin of $\mathrm{BIC}$}

To shed light onto the experimental results shown in Fig. 2, we make use of the coupled electric/magnetic dipole theory (CEMD) for 2D planar arrays developed in Refs. ${ }^{28,29}$ In light of the inhomogeneities of the fabricated meta-atoms (see e.g. Fig. 1c), we have explored a range of disk parameters to properly fit the experimental measurements shown in Fig. 2. For the dielectric function of silicon, we use the measured values by Aspnes and Studna. ${ }^{41}$ We note that we also evaluated the dielectric function of our silicon thin film by using spectroscopic ellipsometry, which gave very similar values. The resulting extinction spectra are shown in Fig. 3 for a square lattice with period $a=340 \mathrm{~nm}$ and disk parameters $h=80 \mathrm{~nm}$ and $D=220 \mathrm{~nm}$; the extinction is calculated as $1-T$, where $T$ is the zerothorder transmitted intensity. This definition of the extinction is equivalent to that in the experiment, i.e., $E=1-T / T_{0}$, because $T_{0}$ is unity in the simulation. The results for $\mathrm{TE}$ 
polarization are shown in Fig. 3a; those for TM polarization are included in the Suppl. Inf. Fig. S2 for the sake of completeness. The in-plane diffraction lines are evident in the CEMD calculations through the abrupt transitions in the extinction spectra. The agreement with the experimental results is remarkable, especially in the long wavelength regions, where only dipolar resonances are excited.

Let us focus on the results for TE polarization shown in Fig. 3a. A weak, low-energy and dispersive band that vanishes upon approaching normal incidence ( $\Gamma$ point), in agreement with the experimental results in Fig. 2a, presents all the features of a BIC, as we will discuss below. Two other broad bands are observed in between $\lambda(\mathrm{nm})=580-650$, which stem from both the in-plane resonances: the dispersive ED resonance $\left(p_{y}\right)$ above $\lambda \sim 620 \mathrm{~nm}$ and the non-dispersive MD resonance $\left(m_{x}\right)$ lying at $\lambda \sim 600 \mathrm{~nm}$. The electric or magnetic characters of the resonances are determined by suppressing the contribution of the other resonances in the CEMD calculations as it is illustrated in the SI (Fig. S3).

Figure 3b displays a CEMD calculation of the extinction for TE polarization, where only the contribution from the vertical MD resonance $\left(m_{z}\right)$ is considered. The most relevant feature in these calculations is the dispersive quasi-BIC. Clearly, the narrowing and vanishing of the band at the $\Gamma$ point reveals the nature of this symmetry-protected BIC, stemming from the fact that the non-degenerate vertical MD resonance is not allowed to emit at normal incidence, which is the only radiation channel imposed by the lattice periodicity and the electromagnetic wavelength. This physical argument is similar to that stated in Ref. ${ }^{29}$ in the $\mathrm{GHz}$ domain. Therein, the high-refractive index of the disk meta-atoms at low frequencies $(\varepsilon \sim 80)$, made it much simpler the experimental observation, since all MD and ED resonances do not overlap.

For the sake of comparison, extinction spectra of the corresponding BIC band resulting from the CEMD calculations (only vertical MD contribution) and the experimental measurements are shown in Figs. 4a and b, respectively, for various angles of incidence. Therein, the extinction spectra are shown in the frequency domain to evidence their spectral line shapes. 
We suppress all contributions other than that from the vertical MD in the CEMD calculations shown in Fig. 4a in order to remove the (small) background that makes the resonance asymmetric, thus allowing us to extract the $Q$-factor of the BIC from the Lorentzian lineshape. Despite the low absorption of polycrystalline Si at $\lambda \sim 750 \mathrm{~nm}$, it has a significant impact on the BIC width, precluding its divergence. As a further proof of the nature of the BIC, we plot in Fig. 4c (inset) the corresponding $Q$-factor as a function of the angle of incidence in the same spectral region as in Fig. 3b but neglecting absorption. This figure neatly reveals the divergence of the $Q$-factor with $Q \gtrsim 10^{4}$ for $\theta_{\text {in }}<2.5^{\circ}$. We include also the $Q$ factor with absorption from the calculations of Fig. $4 \mathrm{a}$, which saturates at $Q \gtrsim 10^{2}$. The $Q$-factor resulting from the measured spectra is also shown in Fig. 4c; as expected, it lies slightly below the maximum value predicted by our CEMD theory with absorption. Still, it is remarkable that such fundamental limit for the $Q$-factor is nearly achieved with a sample that exhibits relatively large inhomogeneities that may lead to radiation loss. ${ }^{34}$ This observation comes in support of the robustness of this symmetry-protected MD BIC.

The effects of absorption and radiative loss on this BIC are further examined by annealing the nanodisk array under $N_{2}$ atomsphere at $1000^{\circ} \mathrm{C}$. This process activates the local diffusion of Si atoms to decrease the number of defect in crystalline Si and thus reduce $k .{ }^{42}$ Meanwhile, the shape of the nanodisks hardly changes because the temperature is still far below the melting point of $\mathrm{Si}\left(=1414^{\circ} \mathrm{C}\right)$. Comparison between the array before and after the annealing (see Fig. 5a) shows that the extinction features notably change with the annealing. The inplane and out-of-plane dipole modes blueshift, because of the reduction of $n$ accompanied by the reduction of $k$ via Kramers-Kronig relation. The BIC is also blueshifted and the $Q$ factors are increased by the heat-treatment (Fig. 5b). It is noted that the values in Fig. 5b are different from those in Fig. 4c, because the samples are different. These samples were prepared using the same design but slightly different etching conditions. The effect is notable especially at longer wavelengths, where the intrinsic absorption of $\mathrm{Si}$ is lower. The dielectric functions before and after the heat-treatment are shown in Supp. Inf. Fig. S5 for the thin 
film, where the treatment indeed reduces both the real and imaginary parts of $\epsilon$. The SEM image in the inset of Fig. 5b indicates the shape of nanodisk does not change and radiation loss still exists after the heat-treatment. Thus the change in the spectral features mainly comes from the reduction of the absorption loss.

For the sake of interest for potential applications, we demonstrate that BICs can be tuned further in the optical domain. Disks with smaller diameters should reduce the resonant frequency on the circulating displacement current and shift the vertical MD resonance towards shorter wavelengths, as experimentally demonstrated in Fig. 6a. This observation is confirmed theoretically through our CEMD for disks of height $h=80 \mathrm{~nm}$ and diameter $D=200$ $\mathrm{nm}$ in Fig. 6b, where the BIC blueshifts to $\lambda \sim 700 \mathrm{~nm}$. The decrease of the BIC to even shorter wavelengths is hindered in our configuration by available disk sizes: if the diameter is further shortened, while keeping the height fixed (limited by the fabrication process used in this study), the aspect ratio is not preserved so that the vertical MD resonances overlaps with other in-plane resonances.

\section{Conclusions}

We have demonstrated experimentally the emergence of symmetry-protected BICs in the optical domain supported by arrays of polycrystalline Si nanodisk. These BICs stem from vertical magnetic dipole resonances. Unlike in high-refractive index disks in the microwave domain, the maximum refractive index contrast attainable in the visible is small $(n \sim 4)$, which is overcome by fabricating flatter disks with low aspect ratio (height/diameter), so that the lowest-order MD resonance parallel to the disk axis barely overlaps with other ED or MD resonances. Our CEMD theory reproduces fairly well the resulting extinction spectra and confirms the symmetry-protected BIC emergence at the $\Gamma$ point with the expected vertical magnetic dipole origin. Despite the relatively large number of inhomogeneities in the sample, $Q$-factors of the order of $10^{2}$ are experimentally achieved, approaching the theoretical limit for 
homogeneous Si disks with finite absorption. These results prove the robustness of the BICs to imperfections and their sensitivity to material absorption. Finally, we have explored the possibility of shifting the BIC frequency towards higher frequencies in the visible through reducing the disk diameter. Indeed, the shift of the BIC is verified within the available geometrical parameters and material losses for this configuration. These results open a new way toward the exploration of optical BICs for light-matter interaction and applications including sensors and lasers. ${ }^{37,43}$ Especially, the application of BICs for strong coupling is promising due to their infinitely-large $Q$ factors in lossless materials. ${ }^{35}$

\section{Methods}

Fabrication. Polycrystalline Si thin films with a thickness of $80 \mathrm{~nm}$ were grown on a synthetic silica glass substrate by low-pressure chemical vapor deposition using $\mathrm{SiH}_{4}$ gas as a source of Si. A resist (NEB22A2, Sumitomo) was cast on the Si film and exposed to electron-beam lithography, followed by development to make nanoparticle arrays of resist on the Si film. The Si film was vertically etched using a selective dry etching (Bosch process) with $\mathrm{SF}_{6}$ and $\mathrm{C}_{4} \mathrm{H}_{8}$ gases, and the resist residue was etched away by oxygen dry etching $\left(\mathrm{O}_{2}\right.$ $100 \mathrm{sccm}, 100 \mathrm{~W}, 10 \mathrm{~Pa}, 1 \mathrm{~min}$.). The fabricated area of the array covers $3 \times 3 \mathrm{~mm}^{2}$.

Rapid thermal annealing. Some of the fabricated Si nanodisk arrays were subjected to rapid thermal annealing (RTA). The samples were heated with a high rate $\left(\sim 20^{\circ} \mathrm{C} / \mathrm{s}\right)$ using halogen lamps to $1000^{\circ} \mathrm{C}$ and kept for 5 min. in $N_{2}$ atmosphere.

Extinction measurements. The nanodisk arrays were in an optically homogeneous environment by placing an upperstrate of fused silica glass with index-matching oil. The samples were placed in a rotational stage, and illuminated by a collimated and polarized beam. The source of illumination was a broadband halogen lamp (Thorlabs SLS201L/M). The beam diameter was $0.7 \mathrm{~mm}$, smaller than the area of the array. The optical axis corresponds to the $z$-axis and the sample was rotated around the $y$-axis (see Fig. 1a). The 


\section{Acknowledgement}

We thank K. Agata and M. Higashino (Kyoto U., Japan) for the RTA and optical measurements. This work was partly supported by the Nanotechnology Hub, Kyoto University and Kitakyusyu FAIS in the "Nanotechnology Platform Project," sponsored by MEXT, Japan. We gratefully acknowledge the financial support from Kakenhi (17KK0133, 19H02434) and the Netherlands Organisation for Scientific Research (NWO) through Gravitation grant "Research Centre for Integrated Nanophotonics" and Innovational Research Activities Scheme (Vici project SCOPE no. 680-47-628). J.A.S.-G. and D.R.A. acknowledge support from 
the Spanish Ministerio de Ciencia e Innovación through grants NANOTOPO FIS201791413-EXP, MELODIA PGC2018-095777-B-C21, and FPU PhD Fellowship FPU15/03566 (MCIU/AEI/FEDER, UE).

\section{Supporting Information Available}

Supporting Information is available: Extinction for the TM-polarized light: Experiment and calculation; Contributions of in- and out-of-plane components to the extinction; BICs in the Si nanodisk array without material absorption; Effect of thermal annealing on dielectric function of Si.

\section{References}

(1) Vaskin, A.; Kolkowski, R.; Koenderink, A. F.; Staude, I. Light-emitting metasurfaces. Nanophotonics 2019, 8, 1151.

(2) Staude, I.; Miroshnichenko, A. E.; Decker, M.; Fofang, N. T.; Liu, S.; Gonzales, E.; Dominguez, J.; Luk, T. S.; Neshev, D. N.; Brener, I.; Kivshar, Y. Tailoring Directional Scattering through Magnetic and Electric Resonances in Subwavelength Silicon Nanodisks. ACS Nano 2013, 7, 7824-7832.

(3) Kuznetsov, A. I.; Miroshnichenko, A. E.; Brongersma, M. L.; Kivshar, Y. S.; Luk'yanchuk, B. Optically Resonant Dielectric Nanostructures. Science 2016, 354, $\operatorname{aag} 2472$.

(4) Staude, I.; Schilling, J. Metamaterial-inspired silicon nanophotonics. Nature Photonics 2017, 11, 274 .

(5) Kerker, M.; Wang, D. S.; Giles, C. L. Electromagnetic scattering by magnetic spheres. J. Opt. Soc. Am. 1983, 73, 765-767. 
(6) García-Cámara, B.; Alcaraz de la Osa, R.; Saiz, J. M.; González, F.; Moreno, F. Directionality in Scattering by Nanoparticles: Kerker's Null-Scattering Conditions Revisited. Opt. Lett. 2011, 36, 728.

(7) Geffrin, J. M.; García-Cámara, B.; Gómez-Medina, R.; Albella, P.; Froufe-Pérez, L. S.; Eyraud, C.; Litman, A.; Vaillon, R.; González, F.; Nieto-Vesperinas, M. Magnetic and Electric Coherence in Forward- and Back-Scattered Electromagnetic Waves by a Single Dielectric Subwavelength Sphere. Nat. Commun. 2012, 3, 1171-1.

(8) Campione, S.; Basilio, L. I.; Warne, L. K.; Sinclair, M. B. Tailoring dielectric resonator geometries for directional scattering and Huygens' metasurfaces. Optics Express 2015, 23, 2293-2307.

(9) Decker, M.; Staude, I.; Falkner, M.; Dominguez, J.; Neshev, D. N.; Brener, I.; Pertsch, T.; Kivshar, Y. S. High-Efficiency Dielectric Huygens' Surfaces. Advanced Optical Materials 2015, 3, 813-820.

(10) Yu, Y. F.; Zhu, A. Y.; Paniagua-Domínguez, R.; Fu, Y. H.; Luk'yanchuk, B.; Kuznetsov, A. I. High-transmission dielectric metasurface with $2 \pi$ phase control at visible wavelengths. Laser $\&$ Photonics Reviews 2015, 9, 412-418.

(11) Babicheva, V. E.; Evlyukhin, A. B. Resonant Lattice Kerker Effect in Metasurfaces With Electric and Magnetic Optical Responses. Laser \& Photonics Reviews 2017, 11, 1700132.

(12) Abujetas, D. R.; Sánchez-Gil, J. A.; Sáenz, J. J. Generalized Brewster effect in highrefractive-index nanorod-based metasurfaces. Opt. Express 2018, 26, 31523.

(13) Castellanos, G. W.; Bai, P.; Rivas, J. G. Lattice resonances in dielectric metasurfaces. Journal of Applied Physics 2019, 125, 213105. 
(14) Castellanos, G. W.; Murai, S.; Raziman, T. V.; Wang, S.; Ramezani, M.; Curto, A. G.; Gómez Rivas, J. Exciton-Polaritons with Magnetic and Electric Character in AllDielectric Metasurfaces. ACS Photonics 2020, \%, 1226-1234.

(15) Murai, S.; Gabriel W. Castellanos, G. W.; Raziman, T.; Curto, A. G.; Gómez Rivas, J. Enhanced light emission by magnetic and electric resonances in dielectric metasurfaces. Adv. Opt. Mater. 2020, 1902024.

(16) Miroshnichenko, A. E.; Evlyukhin, A. B.; Yu, Y. F.; Bakker, R. M.; Chipouline, A.; Kuznetsov, A. I.; Luk'yanchuk, B.; Chichkov, B. N.; Kivshar, Y. S. Nonradiating anapole modes in dielectric nanoparticles. Nature Communications 2015, 6, 8069.

(17) Rybin, M. V.; Koshelev, K. L.; Sadrieva, Z. F.; Samusev, K. B.; Bogdanov, A. A.; Limonov, M. F.; Kivshar, Y. S. High-Q Supercavity Modes in Subwavelength Dielectric Resonators. Physical Review Letters 2017, 119, 243901.

(18) Hsu, C. W.; Zhen, B.; Stone, A. D.; Joannopoulos, J. D.; Soljačić, M. Bound states in the continuum. Nature Reviews Materials 2016, 1, 16048.

(19) Han, S.; Rybin, M. V.; Pitchappa, P.; Srivastava, Y. K.; Kivshar, Y. S.; Singh, R. Guided-Mode Resonances in All-Dielectric Terahertz Metasurfaces. Advanced Optical Materials 2020, 8, 1900959.

(20) Koshelev, K.; Favraud, G.; Bogdanov, A.; Kivshar, Y.; Fratalocchi, A. Nonradiating photonics with resonant dielectric nanostructures. Nanophotonics 2019, 8, 725-745.

(21) Fan, K.; Shadrivov, I. V.; Padilla, W. J. Dynamic bound states in the continuum. Optica 2019, 6, 169-173.

(22) Koshelev, K.; Lepeshov, S.; Liu, M.; Bogdanov, A.; Kivshar, Y. Asymmetric Metasurfaces with High- $Q$ Resonances Governed by Bound States in the Continuum. Physical Review Letters 2018, 121, 193903. 
(28) Abujetas, D. R.; van Hoof, N.; ter Huurne, S.; Gómez Rivas, J.; Sánchez-Gil, J. A. Spectral and temporal evidence of robust photonic bound states in the continuum on terahertz metasurfaces. Optica 2019, 6, 996.

(29) Abujetas, D. R.; Barreda, Á.; Moreno, F.; Sáenz, J. J.; Litman, A.; Geffrin, J.-M.; Sánchez-Gil, J. A. Brewster quasi bound states in the continuum in all-dielectric metasurfaces from single magnetic-dipole resonance meta-atoms. Sci. Rep. 2019, 9, 16048.

(30) Koshelev, K. L.; Sychev, S. K.; Sadrieva, Z. F.; Bogdanov, A. A.; Iorsh, I. V. Strong coupling between excitons in transition metal dichalcogenides and optical bound states in the continuum. Physical Review B 2018, 98, 161113.

(31) Kim, S.; Kim, K.-H.; Cahoon, J. Optical Bound States in the Continuum with Nanowire Geometric Superlattices. Physical Review Letters 2019, 122, 187402. 
(32) Nguyen, T. G.; Ren, G.; Schoenhardt, S.; Knoerzer, M.; Boes, A.; Mitchell, A. Ridge Resonance in Silicon Photonics Harnessing Bound States in the Continuum. Laser \& Photonics Reviews 2019, 13, 1900035.

(33) Han, S.; Cong, L.; Srivastava, Y. K.; Qiang, B.; Rybin, M. V.; Kumar, A.; Jain, R.; Lim, W. X.; Achanta, V. G.; Prabhu, S. S.; Wang, Q. J.; Kivshar, Y. S.; Singh, R. All-Dielectric Active Terahertz Photonics Driven by Bound States in the Continuum. Advanced Materials 2019, 31, 1901921.

(34) Sadrieva, Z. F.; Sinev, I. S.; Koshelev, K. L.; Samusev, A.; Iorsh, I. V.; Takayama, O.; Malureanu, R.; Bogdanov, A. A.; Lavrinenko, A. V. Transition from Optical Bound States in the Continuum to Leaky Resonances: Role of Substrate and Roughness. ACS Photonics 2017, 4, 723-727.

(35) Kolodny, S.; Iorsh, I. Q/V enhancement of micropillar resonator in bound states in the continuum regime. Optics Letters 2020, 45, 181-183.

(36) Yesilkoy, F.; Arvelo, E. R.; Jahani, Y.; Liu, M.; Tittl, A.; Cevher, V.; Kivshar, Y.; Altug, H. Ultrasensitive hyperspectral imaging and biodetection enabled by dielectric metasurfaces. Nature Photonics 2019, 13, 390-396.

(37) Ha, S. T.; Fu, Y. H.; Emani, N. K.; Pan, Z.; Bakker, R. M.; Paniagua-Domínguez, R.; Kuznetsov, A. I. Directional lasing in resonant semiconductor nanoantenna arrays. $\mathrm{Na}$ ture Nanotechnology 2018, 13, 1042-1047.

(38) Matsubara, H.; Yoshimoto, S.; Saito, H.; Jianglin, Y.; Tanaka, Y.; Noda, S. GaN Photonic-Crystal Surface-Emitting Laser at Blue-Violet Wavelengths. Science 2008, $319,445-447$.

(39) Lee, J.; Zhen, B.; Chua, S.-L.; Qiu, W.; Joannopoulos, J. D.; Soljačić, M.; Shapira, O. Observation and Differentiation of Unique High- $Q$ Optical Resonances Near Zero Wave 
Vector in Macroscopic Photonic Crystal Slabs. Physical Review Letters 2012, 109, 067401 .

(40) Doeleman, H. M.; Monticone, F.; den Hollander, W.; Alù, A.; Koenderink, A. F. Experimental observation of a polarization vortex at an optical bound state in the continuum. Nature Photonics 2018, 12, 397-401.

(41) Aspnes, D. E.; Studna, A. A. Dielectric functions and optical parameters of Si, Ge, GaP, GaAs, GaSb, InP, InAs, and InSb from 1.5 to 6.0 eV. Physical Review B $\mathbf{1 9 8 3 ,}$ 27, 985-1009.

(42) Zhang, F.; Proust, J.; Gérard, D.; Plain, J.; Martin, J. Reduction of Plasmon Damping in Aluminum Nanoparticles with Rapid Thermal Annealing. The Journal of Physical Chemistry C 2017, 121, 7429-7434.

(43) Wu, M.; Ha, S. T.; Shendre, S.; Durmusoglu, E. G.; Koh, W.-K.; Abujetas, D. R.; Sánchez-Gil, J. A.; Paniagua-Dominguez, R.; Demir, H. V.; Kuznetsov, A. I. RoomTemperature Lasing in Colloidal Nanoplatelets via Mie-Resonant Bound States in the Continuum. Nano Letters 2020, XX, XXXX.

(44) Reid, M. H.; Johnson, S. G. Efficient Computation of Power, Force, and Torque in BEM Scattering Calculations. arXiv:1307.2966

(45) Pierce, D. T.; Spic er, W. E. Electronic Structure of Amorphous Si from Photoemission and Optical Studies. Physical Review B 1972, 5, 3017-3029.

(46) Puychevrier, N.; Menoret, M. Synthesis of III-V semiconductor nitrides by reactive cathodic sputtering. Thin Solid Films 1976, 36, 141-145.

(47) Tyagai, V.; Evstigneev, A.; Krasiko, A., A.N.and Andreeva; Malakhov, V. Optical properties of indium nitride films. Sov. Phys. Semicond. 1977, 11, 1157-1159. 
(48) Luke, K.; Okawachi, Y.; Lamont, M. R. E.; Gaeta, M. A. L.; Lipson, M. Broadband mid-infrared frequency comb generation in a $\mathrm{Si}_{3} \mathrm{~N}_{4}$ microresonator. Opt. Lett. 2015, 40, 4823-4826.

(49) Cambiasso, J.; Grinblat, G.; Li, Y.; Rakovich, A.; Cortés, E.; Maier, S. A. Bridging the Gap between Dielectric Nanophotonics and the Visible Regime with Effectively Lossless Gallium Phosphide Antennas. Nano Letters 2017, 17, 1219-1225.

(50) Lin, M.; Sverdlov, B. N.; Strite, S.; Morkoç, H.; Drakin, A. E. Refractive indices of wurtzite and zincblende GaN. Electronics Letters 1993, 29, 1759-1760. 


\section{Graphical TOC Entry}

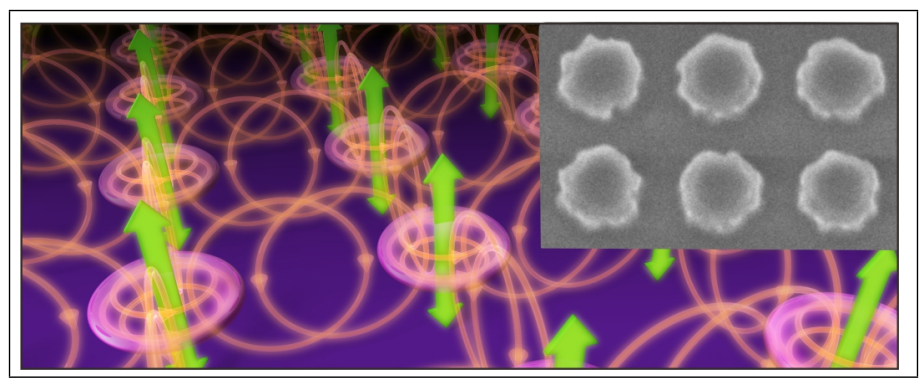



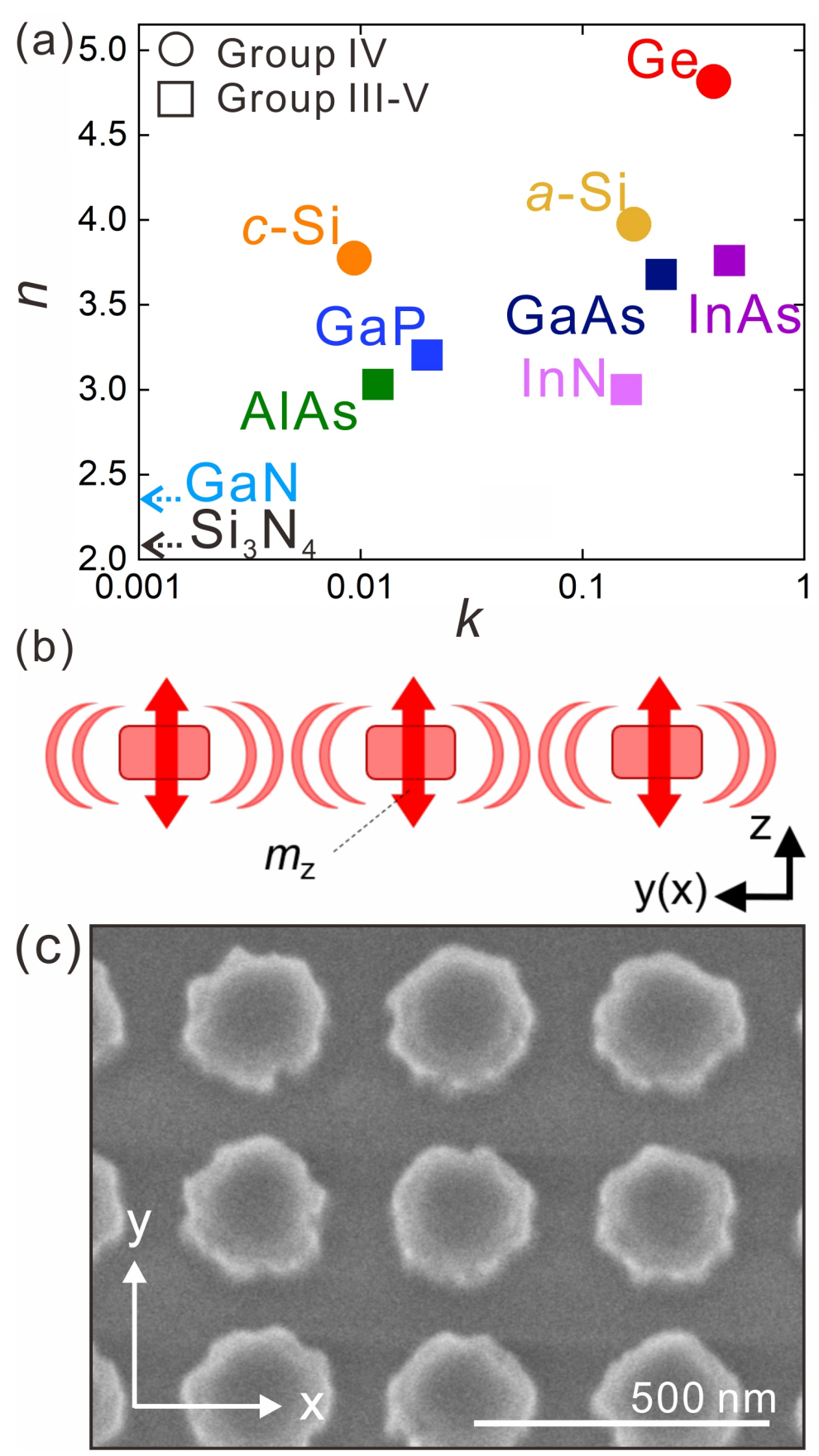

Figure 1: (a) Refractive index $(n)$ and extinction coefficient $(k)$ of high-refractive-index dielectric materials at $\lambda=750 \mathrm{~nm}$ : Ge ${ }^{41}$ amorphous $\mathrm{Si}(a-\mathrm{Si}),{ }^{45}$ crystalline $\mathrm{Si}(c-\mathrm{Si}),{ }^{41}$ InAs ${ }^{41} \mathrm{InN},{ }^{46,47} \mathrm{GaAs},{ }^{41} \mathrm{Si}_{3} \mathrm{~N}_{4},{ }^{48} \mathrm{GaP}^{49}$ and GaN. ${ }^{50}$ Both GaN and $\mathrm{Si}_{3} \mathrm{~N}_{4}$ are virtually transparent at $\lambda=750 \mathrm{~nm}$ and the $n$ values are denoted as arrows at the edge of the plot. (b) Design concept of the vertical MD BICs. A sub-diffraction lattice of silicon nanodisk can support out-of-plane magnetic resonances which decay into the lateral directions. This mode is symmetry-protected and cannot be excited by normal incidence. (c) SEM image of the Si nanodisk array. The coordinate axes are also indicated. 

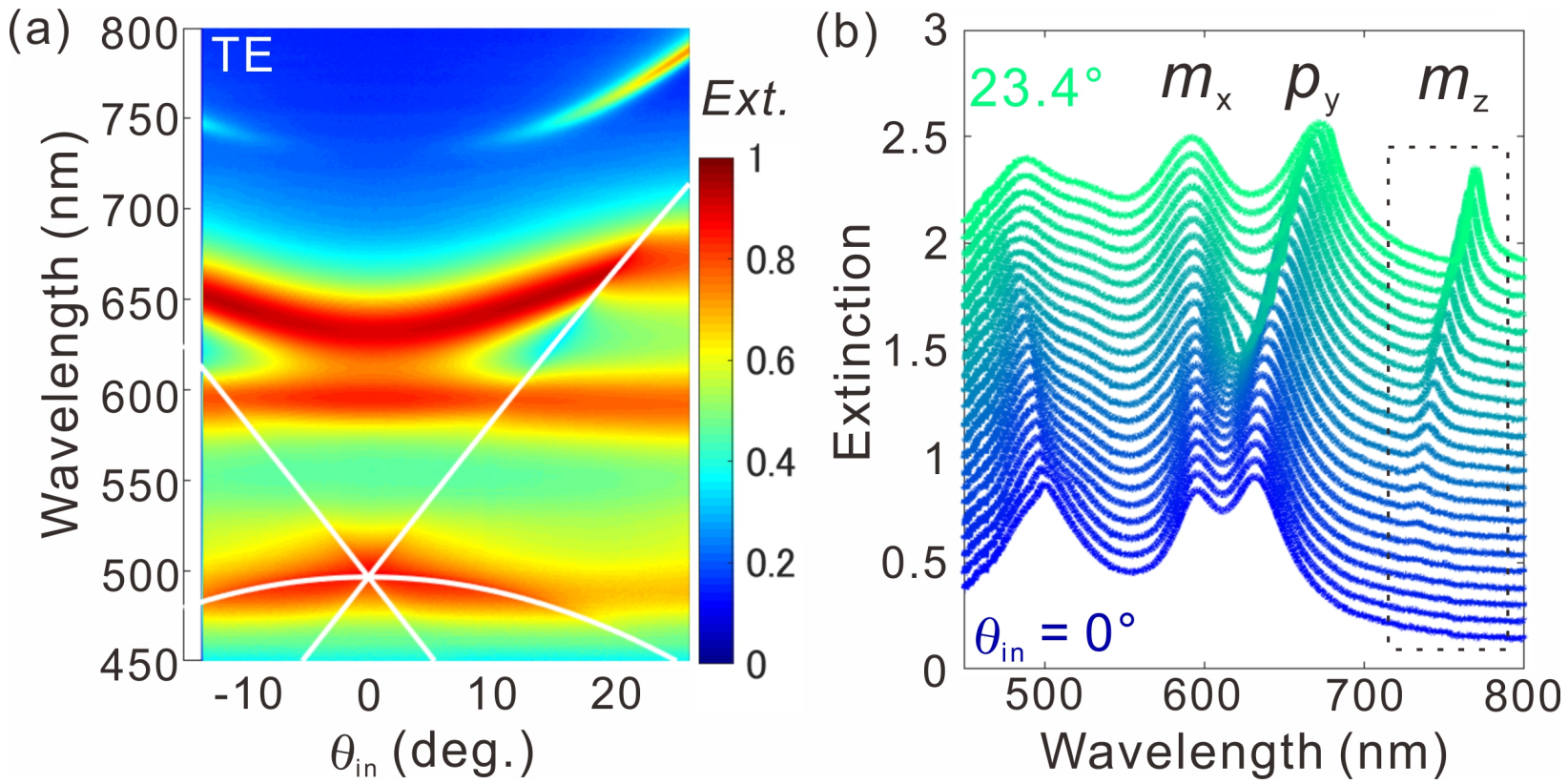

Figure 2: (a) Experimental extinction of the nanodisk array (period $=340$, height $=80$ and diameter $=248 \mathrm{~nm}$ ) for TE-polarized light. (b) Cuts of the extinction with varied angle of incidence for TE-polarized light. The spectra are shifted vertically for the sake of clarity. The dotted square denotes the spectral region where the BIC occurs.
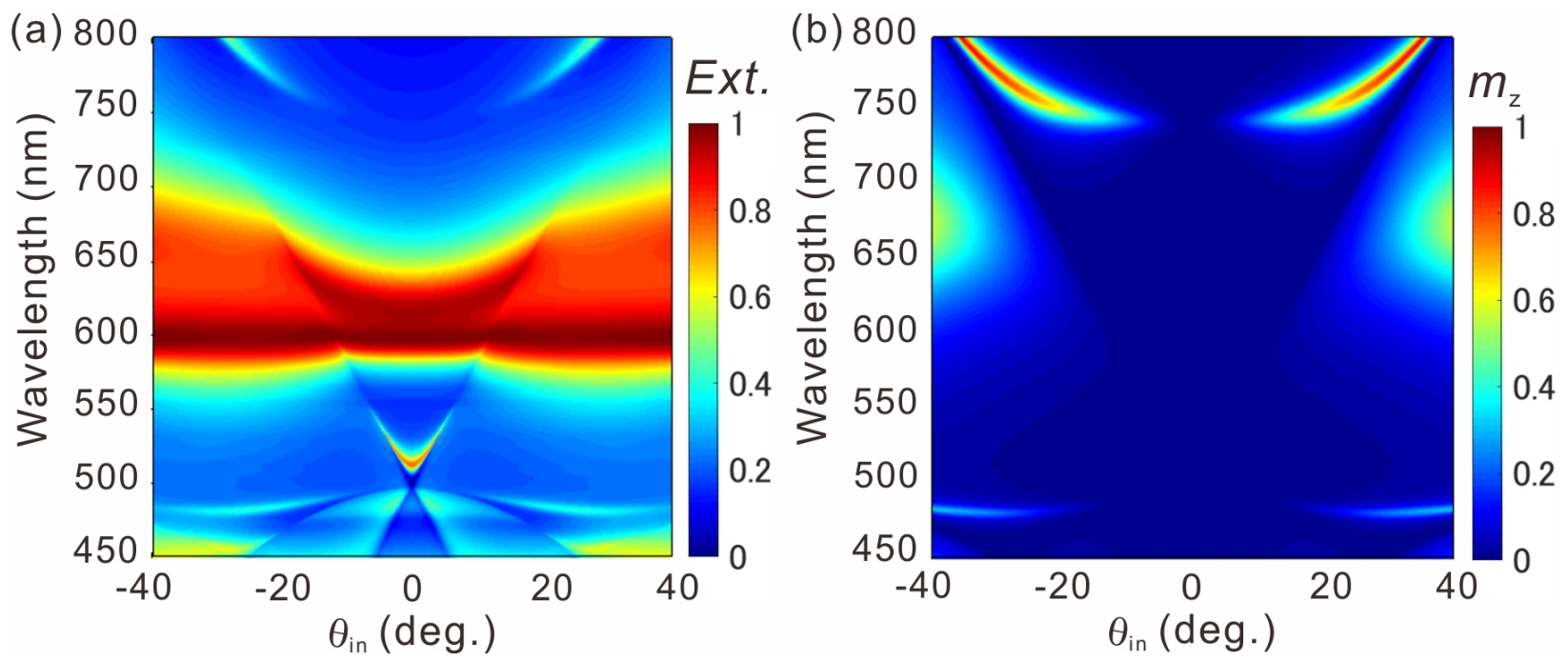

Figure 3: (a) Calculated (CEMD) extinction of the nanodisk array for TE-polarized light. (b) Contributions from the out-of-plane components of the electric and magnetic dipole resonances for TE-polarized light. 

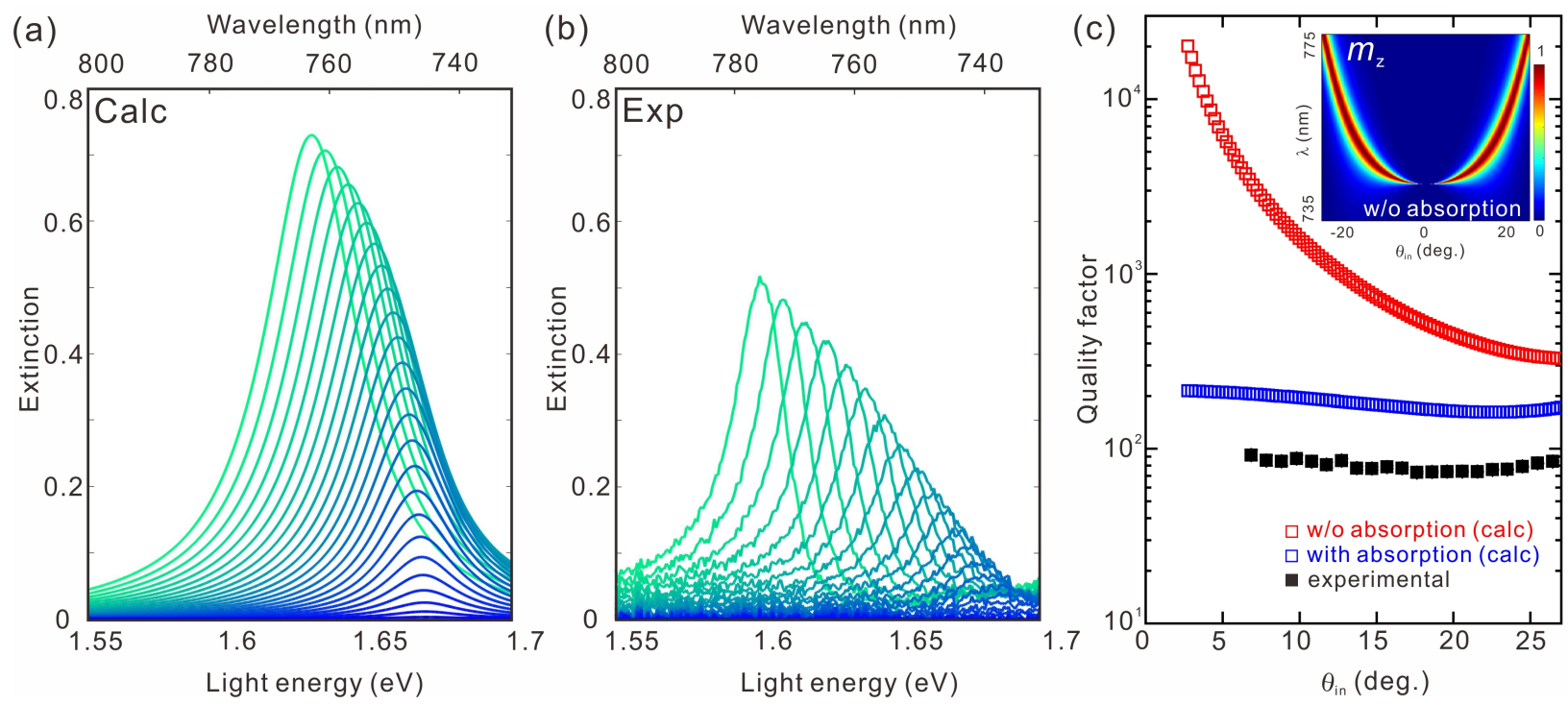

Figure 4: Extinction spectra for various angles of incidence in the vicinity of the BIC band from Fig. 3a (a, theoretical CEMD calculations, including only the out-of-plane MD contribution), and from Fig. 2a in (b, experimental). $\theta_{i n}=0$ to $25^{\circ}$ in $1.0^{\circ}$ steps. The experimental extinctions are shown after subtracting the spectrum at $\theta_{i n}=0^{\circ}$ where no spectral feature appears in the range of interest, to eliminate the experimental background. Comparison of $Q$ factors of quasi-BIC mode extracted from the extinction for TE polarized light. (c) Calculated (CEMD) $Q$ factors with (represented as blue symbols) and without absorption (red), as well as experimentally obtained $Q$ factors (black) as a function of $\theta_{i n}$. The values from the calculation, properly extracted from the Lorentzian fit of the contribution of the out-of-plane MD quasi-BIC, are however multiplied by two in order to make them directly comparable with the experimental $Q$-factors, which exhibit a spectral Fano lineshape as a result of the interference of such quasi-BIC with the background of the in-plane modes, thus reducing their full width at half maximum by half. 

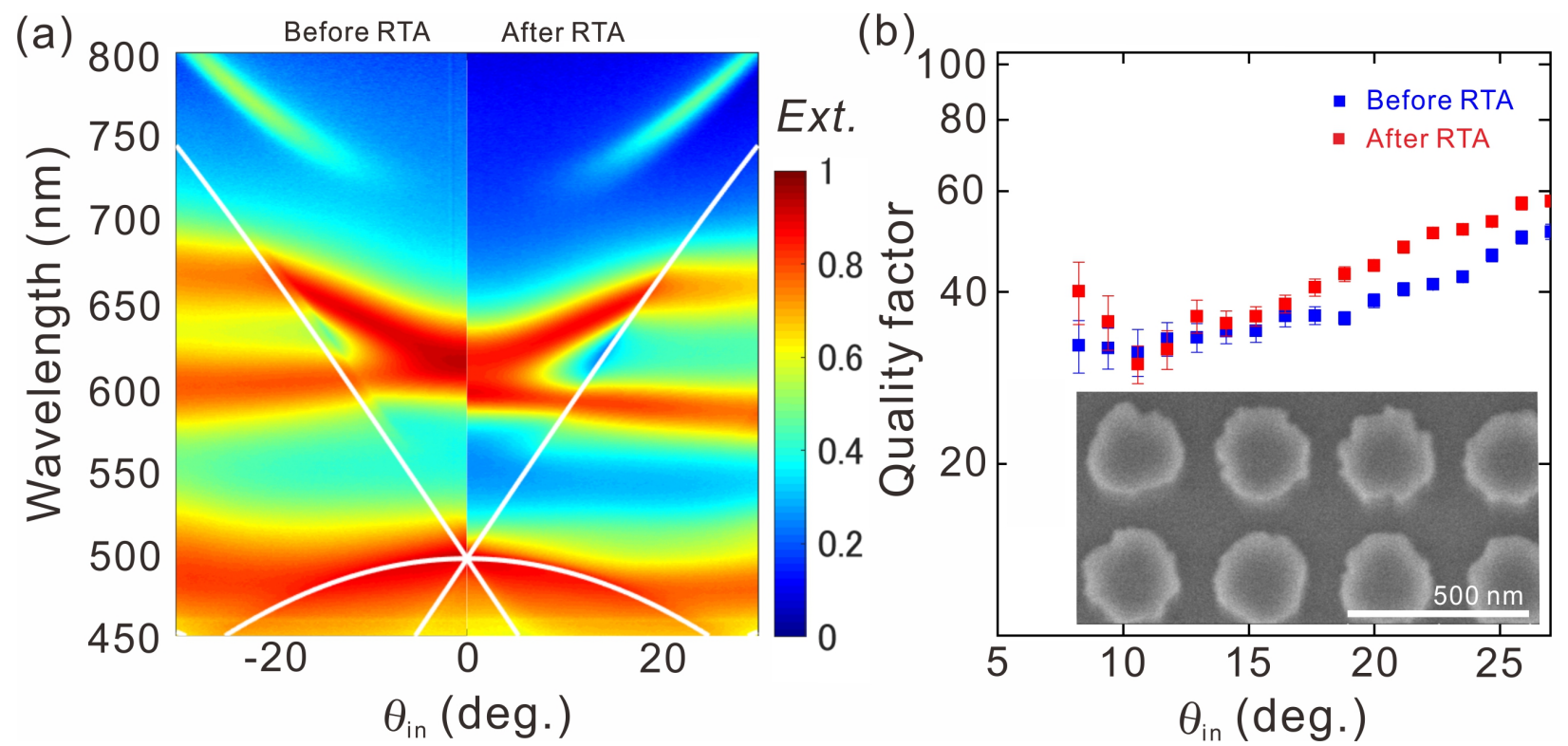

Figure 5: (a) Experimental extinction of the nanodisk array for TE light, before and after the rapid thermal annealing (RTA) in $N_{2}$ at $1000{ }^{\circ} \mathrm{C}$. (b) $Q$ factors of the quasi-BIC mode extracted from the extinction before and after RTA. Inset shows the top-view SEM image after RTA. Note that the $Q$ factor values are different from those on Fig. 4c. They are the samples prepared using the same design but slightly different etching conditions.
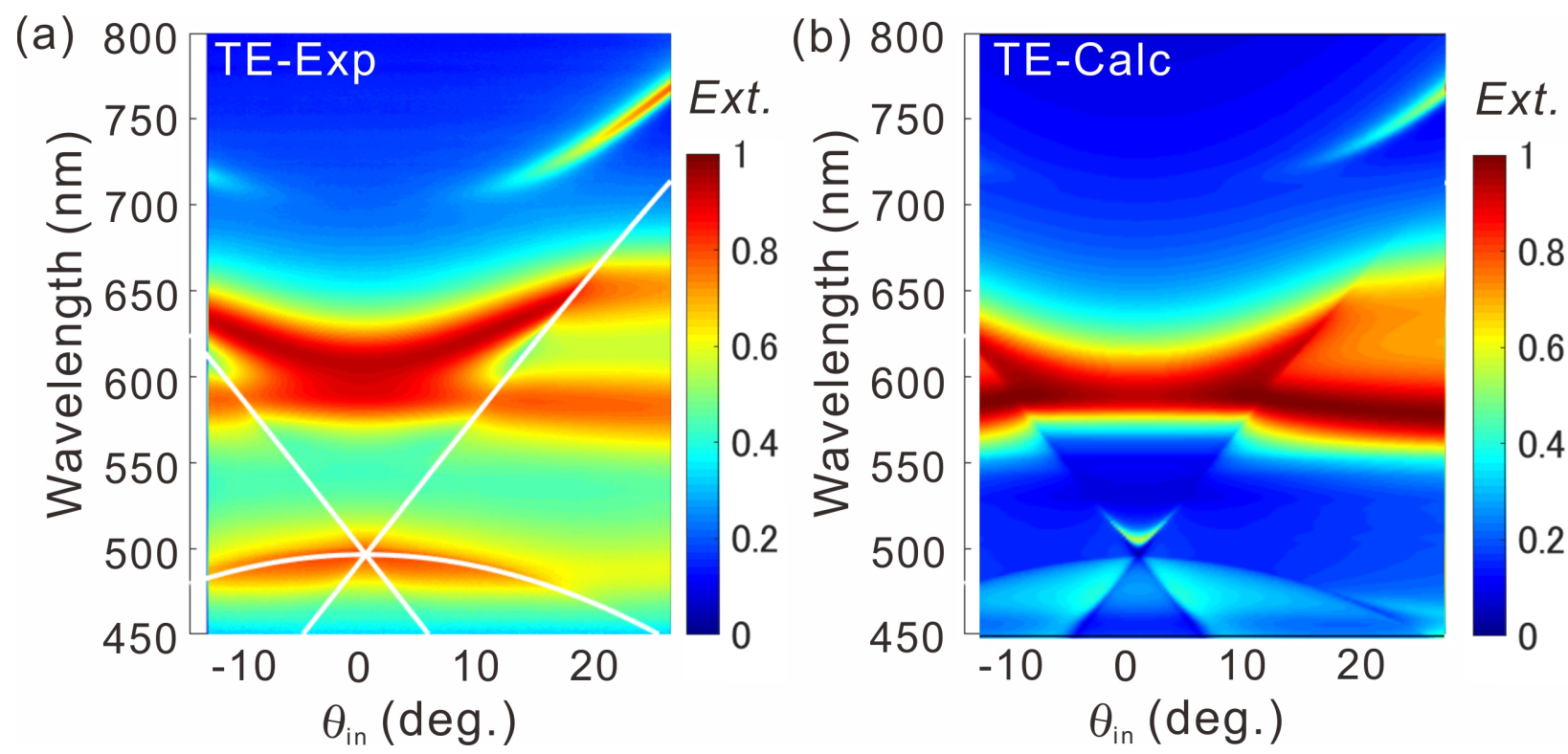

Figure 6: (a) Experimental extinction of the nanodisk array consisting of smaller nanodisks (period $=340$, height $=80$ and diameter $=226 \mathrm{~nm}$ ) for TE-polarized light. (b) Calculated (CEMD) extinction of the nanodisk array for TE-polarized light. 